\title{
Unsupervised Learning based Degradation Estimation and Abnormal Working Condition Detection for a Class of Anthropomorphic Robotic Hand
}

\author{
Carlos F. Cortés Mac-Evoy ${ }^{1}$, Jonathan Manrique Garay ${ }^{2}$, Frank Kirchner ${ }^{3}$ \\ ${ }^{1}$ Universität Bremen, Bremen, Bremen, 28359, Germany \\ cortesmc@uni-bremen.de \\ ${ }^{2}$ Otto-von-Guericke Universität Magdeburg, Magdeburg, Sachsen-Anhalt, 39106, Germany \\ jonathan.manrique@ovgu.de \\ ${ }^{3}$ DFKI Bremen, Bremen, Bremen, 28359, Germany \\ frank.kirchner@dfki.de
}

\begin{abstract}
Grasping is one of the most common tasks related to robotics and manipulation which has received an extensive amount of contributions from the research community. From a design point of view, the robotic gripper systems are generally manufactured using a significant amount of small moving parts, in order to establish a balance between size, weight and performance. This balance leads to designs and components that are less robust than those of, for example, pneumatic grippers. To the best of our knowledge, most of the literature related to robotic grasping concentrates and focuses on grasping from a cognitive perspective. However, in order to ensure the execution of grasping tasks over extended periods of time, reducing down times and increasing gripper availability, even in demanding scenarios without access to maintenance, other phenomena such as component tear and degradation have to be monitored and analyzed. This paper proposes an unsupervised learning model based approach for the estimation of the degradation states and the detection of abnormal working conditions of the actuator components for a class of robotic anthropomorphic hand. The approach allows an easy implementation and establishes the basis for the development of remaining useful life estimation algorithms for the components of other gripper systems. Our proposed architecture consists of an automatic degradation estimator and working condition detector, based on an unsupervised model combining $\mathrm{K}$ means with a geometric approach. The model estimates the hand's actuators degradation and determines its working condition from the online data collected during grasping tasks. The proposed method is able to work both online and of-

\footnotetext{
Carlos F. Cortés Mac-Evoy et al. This is an open-access article distributed under the terms of the Creative Commons Attribution 3.0 United States License, which permits unrestricted use, distribution, and reproduction in any
} medium, provided the original author and source are credited.
\end{abstract}

fline, requires a low amount of data and is independent of the grasped object properties such as mass, geometry, shape, etc. It was experimentally tested on a real Schunk SVH Hand used to assist humans during the assembly process in the automobile industry.

\section{INTRODUCTION}

The phenomenon of the degradation is of central importance in condition based monitoring (CBM) and prognostics and health management (PHM), as it provides engineers, practitioners and scientists crucial information in order to determine the current health status of a component or equipment and therefore, plan the necessary actions for maintenance, inspection or repair of degraded systems, aiming to improving the maintenance schedule, reducing maintenance costs and extending the lifetime of the systems and equipment (Huynh, Grall, \& Berenguer, 2019; Walck, Haschke, Meier, \& Ritter, 2017; Lei, Li, \& Lin, 2016).

In the literature, the approaches for the degradation estimation can be grouped in two families: first, the model based approaches, which attempt to reconstruct the dynamics of the deterioration by means of a physical model, i.e. crack growth, fatigue or wear dynamics. Given the mathematical and system's knowledge required to represent the physical degradation, the Wiener process is one of the most popular tools for modeling the deterioration of a system. Generally the physical models have a limited applicability due to the occurrence of multiple and non linear degradation processes on the operating assets (Loutas, Roulias, \& Georgoulas, 2013). (Wang, $\mathrm{Hu}, \&$ Fan, 2018) propose a deterioration model with a non linear variability that takes into account the cumulative effect of the degradation in the system. The model is then used to determine the Remaining Useful Life (RUL) with application 
to bearings. (Lei et al., 2016) propose a stochastic model with 4 variability sources in combination with a particle filter algorithm to estimate the degradation states for subsequent RUL estimation of machinery. (Huynh et al., 2019) develops a new parametric stochastic framework for maintenance decisions that uses the Gamma process for the degradation modeling. (Garay \& Diedrich, 2019) proposes a linear model for the estimation of the degradation of a glue machine in the automotive industry, enabling the prediction of faults weeks before their occurrence. In a similar way, (Zheng, 2019) estimates the degradation of bearings using a linear model and the Hilbert Entropy.

The second family of approaches are the data-driven methods, which are based upon the system's historical collected data in order to construct a representation of the degradation. These approaches are mostly based on machine learning techniques, including supervised and unsupervised learning. (Khelif et al., 2017) and (Patil et al., 2015) propose a Support Vector Machine (SVM) model used as regressor for the degradation estimation of turbines and batteries respectively. (Loutas et al., 2013) propose a probabilistic Support Vector Regression framework that determines a bounded RUL of bearings based on vibration signals. Their method allows the implementation of general purpose prognostics frameworks using limited or small amount of historical data. (Tobon-Mejia, Medjaher, Zerhouni, \& Tripot, 2012) propose a Mixture of Gaussian Markov Hidden Model (MoG-HMM) to compute the best fit across the degradation phenomenon of ball bearings. (Rodrigues, 2018) obtains the degradation state of each individual component of a multiple component system based on particle filters. (Peng, Cheng, Qiao, \& Qu, 2017) uses a recurrent neural network (RNN) to estimate the future health states of a gearbox attached using noise-to-signal ratio as current health state indicator.

From the above introduction, it is clear that there exists a vast amount of research regarding degradation estimation of single components. Most of the literature focuses on the deterioration estimation of systems and components than can be directly measured, i.e bearings, turbofans, batteries, pumps, etc. However, the estimation of the degradation phenomenon of multiple components is much more challenging (Rodrigues, 2018). In robotic grasping, gripper systems are built using a significant amount of small components, i.e. spindles, small gearboxes, couplings, pulleys, tendons, etc. in order to keep a certain equilibrium between performance and size (Walker, De La Rosa, Elias, Godden, \& Goldsmith, 2010), leading to a very limited amount of sensor data for building a representation of the degradation phenomenon. Furthermore, according to the best of our knowledge, most of the literature related to robotic grasping concentrates and focuses on grasping from a robot-centric cognitive perspective. However, in order to ensure the execution of grasping tasks over extended periods of time in demanding scenarios without ac- cess to maintenance (such as the outer space, deep sea or hazardous environments), the degradation states of the gripper systems have to be taken into account. In our knowledge, this problem has been partially tackled by (Walck et al., 2017) with their implementation of a force limiting algorithm that imitates the human muscle fatigue dynamics to protect the tendons of an anthropomorphic hand from being cut off due to high long-term tensions during grasping, allowing the proper functioning of the hand for more than 3 years without tendon breakage. Also, (Suehiro, Ozawa, \& Van Heerden, 2017) propose a model based switching control architecture to cope with tendon breakage for redundant tendon-drivenmechanisms (TDM) in a fault-tolerant fashion.

This paper attempts to tackle and contribute to the problem of the degradation estimation on a real robotic hand that comprises multiple small and immeasurable components. Our approach is based on an unsupervised learning model that takes advantage of the $K$-means algorithm to estimate the degradation states of the fingers of the robotic hand. Upon these estimations, our approach also provides an operation condition indicator based on predefined degradation thresholds.

The remainder of this paper is organized as follows: Section 2 describes the justification for the development of the proposed method. The section 3 describes the system under study. Section 4 presents the methodology followed for the development of our approach. The sections 5 through 6 provide the experimental results of our method tested on a real robotic hand and other aspects such as parameter tuning and limitations of the method. Lastly, in section 7 brief concluding remarks are presented.

\section{BACKGROUND AND MOTIVATION}

The degradation phenomenon has been studied by several authors (Lei et al., 2016; Si, Zhang, \& Hu, 2017; Garay \& Diedrich, 2019) and can be modeled by a Wiener process as follows:

$$
X(t)=X(0)+\theta(t) t^{b}+\sigma B(t)+\epsilon(t)
$$

where $X(0)$ corresponds to the initial degradation. $\theta(t) t^{b}, b \geq 0$ is called the non linear variability with $\theta \sim N\left(\mu_{\theta}, \sigma_{\theta}\right), \sigma B(t)$ is temporal variability with $\sigma B \sim N\left(0, \sigma^{2} t\right)$ and $B(t)$ is a Brownian motion. Lastly, $\epsilon(t)$ is the random measurement error. Some authors, such as (Zheng, 2019) and (Garay \& Diedrich, 2019), use $b=1$, which turns the model of Eq. (1) into a linear model linear as:

$$
X(t)=X_{0}+\theta(t) t+\sigma B(t)
$$

however, the mechanical systems generally exhibit non linear degradation dynamics (Lei et al., 2016). Also, there exists a convention around the initial degradation in where $X(0)=0$ 
(Wang et al., 2018; Si et al., 2017; Lei et al., 2016; Huynh et al., 2019). In the case of our robotic hand, this assumption cannot be considered, since our conducted studies were performed after some time the hand had been in operation, therefore $X(0)>0$. Note here, that the cumulative effect of the degradation is not being taking into account in Eq. (1). Another difficulty with the models given by Eqs. (1) and (2) is the parameter initialization, since in our case the fingers of the hand incorporate multiple immeasurable components interacting together, such as spindles, gearboxes and couplings, therefore it is not possible to choose the right parameters. The high complexity of such an engineering system would require a degradation model for each one of the components. In other words:

$$
X_{\mathrm{Net}}(t)=\sum_{i=1}^{n}\left(X_{i}(0)+\theta_{i}(t) t^{b_{i}}+\sigma_{i} B_{i}(t)+\epsilon_{i}(t)\right)
$$

which makes its applicability to our particular system unfeasible.

Despite these difficulties several authors have been able to overcome the drawbacks of the model of Eq. (1) by using machine learning techniques, such as Support Vector machines (Loutas et al., 2013; Khelif et al., 2017; Patil et al., 2015), Gaussian Mixture Hidden Markov Models (Tobon-Mejia et al., 2012) and neural networks (Peng et al., 2017), however their approaches are based on well known existing datasets and therefore, their applicability is restricted to the amount of historical data available, which is generally hard to obtain (Ramasso \& Gouriveau, 2014; Garay \& Diedrich, 2019).

\section{Experimental Setup}

\subsection{The Robotic Hand Under Study}

The anthropomorphic hand used in our experiments corresponds to a Schunk SVH 5-fingered Hand which is able to provide sensor data in terms of joint angular positions and motor currents. The hand is driven by 9 motors actuating 20 joints (Ficuciello, 2019) by means of lead screw mechanisms that convert prismatic motion into rotational motion (Ruehl et al., 2014). A description of the nomenclature of this hand is presented in Figure 1. The hand is controlled by a position controller and a current controller working in cascade that are interfaced via a driver compatible with the Robot Operating System (ROS). Since there aren't built-in finger tip force sensors available on this hand, the grasping is performed by a grasp controller actuating in motor space based on the motor torque measurements and therefore, it is only possible to determine the enclosure of an object (Kappassov, Corrales, \& Perdereau, 2015). Figure 1 depicts the nomenclature convention used for the hand and the system's architecture is depicted in Figure 2. For further details regarding the soft- ware and hardware related to the hand, readers are referred to (Schunk Hand SVH Assembly and Operating Manual, Last access: 2020-05-16 00:56:44) and (ROS Driver, Last access: 2020-05-16 01:02:10).

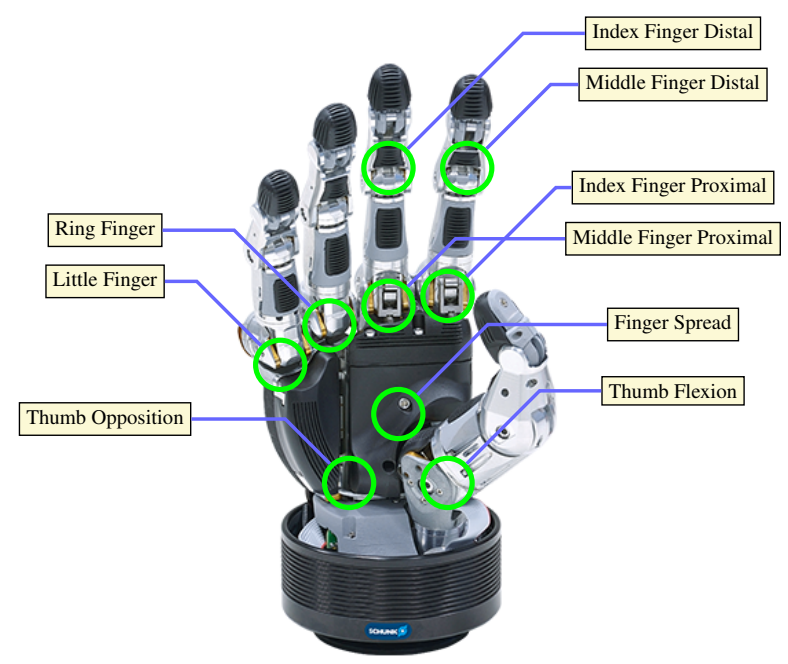

Figure 1. Schunk Hand SVH Nomenclature

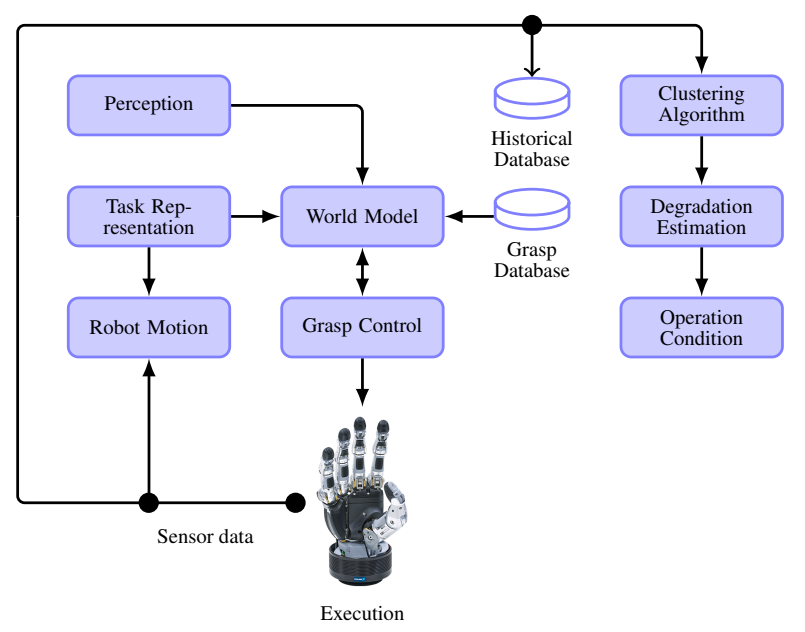

Figure 2. Diagram of the architecture

\subsection{Training Data}

The hand was set to perform a free movement on a regular basis: once a day during ten minutes. During this time both the position and motor current signals of each motor of the hand were recorded using ROS at a frequency of $100 \mathrm{~Hz}$ and afterwards stored on a local database. Given the limited variety of available sensor data, the free movement was chosen to be a free open-close movement across the mechanical movement 
range of all the fingers of the hand in order to isolate it from external disturbances and loads and have a better picture of the actual system's behavior. Otherwise, the measurements would be influenced by the effects of the loads, disturbances and other phenomena like heat transfer, deformation, etc. The Figure 3 show the angular position and its corresponding motor current signal for a specific finger.

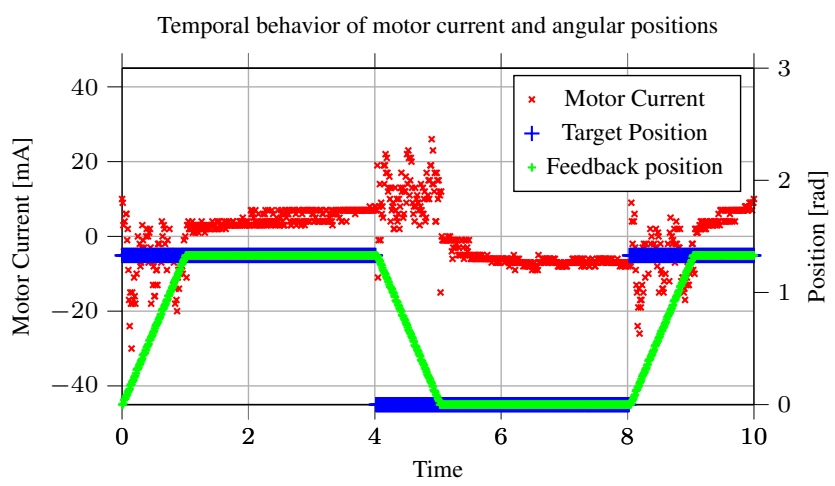

Figure 3. Measured signals on the interval $[0,10]$

From these experiments, several statistical parameters were calculated per day as well as using a moving window of 1000 records, such as the mean $\mu$, variance $\sigma$, Skewness $S$, Kurtosis $K$. Some of the features are summarized in Table 1

\begin{tabular}{|c|c|}
\hline Root Mean Square & $\sqrt{\frac{1}{n} \sum_{i=1}^{n} x_{i}^{2}}$ \\
\hline Variance & $\sigma=\sum_{i=1}^{n} \frac{\left(x_{i}-\mu\right)^{2}}{n}$ \\
\hline Skewness & $S=\sum_{i=1}^{n} \frac{\left(x_{i}-\mu\right)^{3}}{\sigma^{3}}$ \\
\hline Kurtosis & $K=\sum_{i=1}^{n} \frac{\left(x_{i}-\mu\right)^{4}}{\sigma^{4}}$ \\
\hline
\end{tabular}

Table 1. Time-domain features

The aforementioned experiment has been conducted for more than 50 days for every of all the 9 motors of the hand, which leads to a dataset size of about 27 million records. The whole data set consists primarily of the measurable signals such as motor current, angular position and desired angular position, and a set of features are computed and inserted in a separate process after the experiment. It is important to note here, that during the course of each individual experiment it is not possible to classify the actual run as normal or abnormal at first glance, because the target positions were always reached by the fingers. The inspection of the current consumption of each motor indicates whether the finger is working under abnormal conditions. The set of features, include among others, the ones described in Table 1 and the structure of the dataset is shown in Figure 4, where $1 \leq E_{d} \leq 53,1 \leq i \leq 9$ and $j=1$ or $j=2$, depending on the finger.

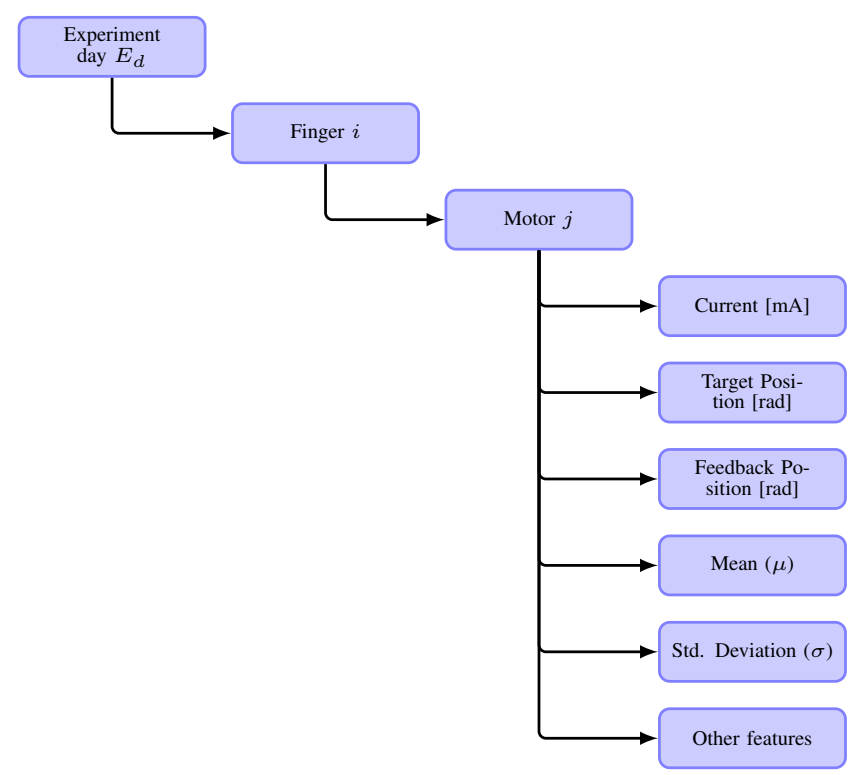

Figure 4. Dataset structure

\section{Methodology}

\subsection{Observed Dynamics of the Measured Signals}

On healthy fingers, the motor current is mostly concentrated on a whole single data unit or cluster across the corresponding mechanical movement range in absence of loads (grasping). However, as the fingers degrade over time, it is observed that their associated motor currents begin to split and form additional clusters, whose distance increases over time. All the motors of the hand's fingers exhibit the same dynamic behavior during free motion in healthy conditions. When they are traveling to the closed position (fist), the motor currents are negative and positive when traveling back. On a healthy fingers the distance between these clusters is small. This can be visualized in Figures 5 and 6.

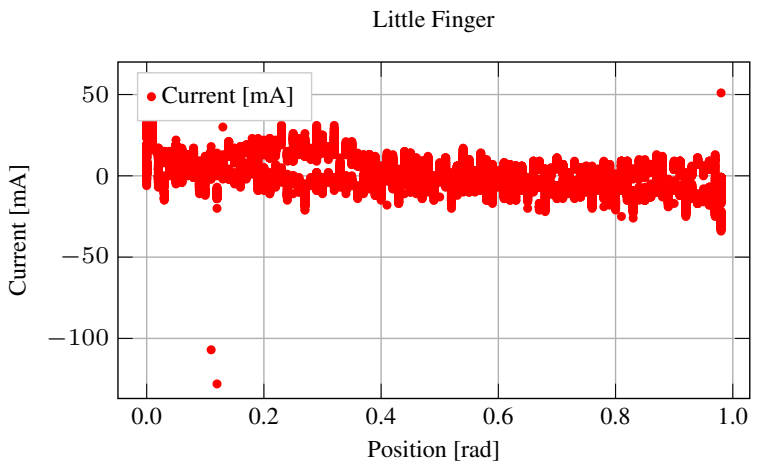

Figure 5. Behavior of the current signal of a healthy finger during free motion 


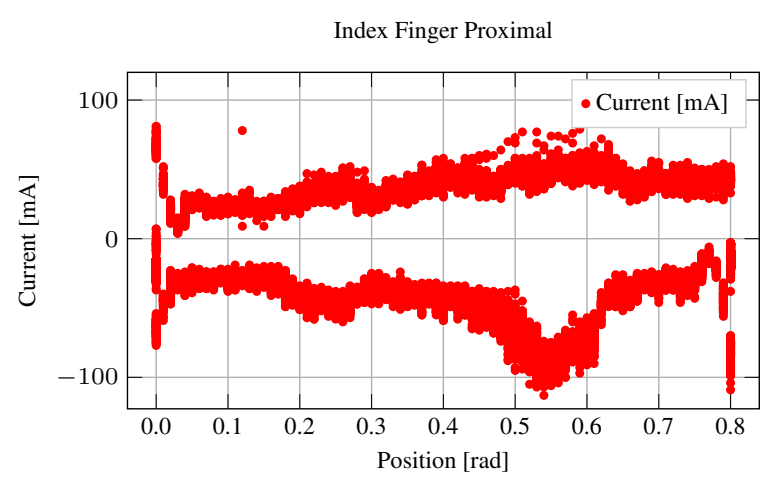

Figure 6. Behavior of current signal of an unhealthy finger during free motion

\subsection{Proposed Method}

Based on the previous observation, we trained an unsupervised learning model based on $K$-Means to analyze the evolution of the clusters over time. The angular position $\theta^{1}$, motor currents $\mathbf{C}_{\mathbf{m}}$ and their corresponding spectrum frequencies $\mathbf{C}_{\mathbf{m f}}$ were used as features for the models, since they were the signals that revealed most information about the degradation. It is important to note that, even though the angular velocity of the fingers could be analytically determined in absence of velocity sensors, this signal actually covers up some information about the underlying phenomena. The $K$-Means algorithm determines the centroids of the clusters in the data and based on the position of the centroids, the euclidean distance between them is computed by

$$
D_{i j k}=\sqrt{X_{i j k}^{2}+Y_{i j k}^{2}}
$$

where $i, j, k>0, i \neq j, j>i$ and $X_{i j k}=\left(x_{i k}-\right.$ $\left.x_{j k}\right) ; \quad Y_{i j k}=\left(y_{i k}-y_{j k}\right)$. A vector $\mathbf{D} \in \mathbb{R}^{k \times p}$ contains all the distances between the clusters centroids across the entire database, with $k=1, \ldots, 53, p=n(n-1) / 2$ and $n>2$ being the number of clusters.

Several parameters such as the sum, $\min , \max , \mu, \sigma$ as well as the area covered by the centroids, were calculated from vector $\mathbf{D}$, in order to deal with the classification errors produced by the $K$-means algorithm and also to be able to construct the estimated degradation index.

According to (Loutas et al., 2013), a good condition based monitoring descriptor, in this case the estimated degradation index, must have a monotonic behavior and a low volatility. These measures were determined using the Spearman's Rank Correlation coefficient and the standard deviation $\sigma$ over the previous parameters respectively.

The Figure 7 shows that the minimum distance between the clusters' centroids given by $\min _{i, j}\left\{\mathbf{D}_{\mathbf{i j}} \in \mathbb{R}^{p \times 1}\right\}$ exhibits

\footnotetext{
${ }^{1}$ Not to be confused with $\theta(t)$ in Eq. (1)
}

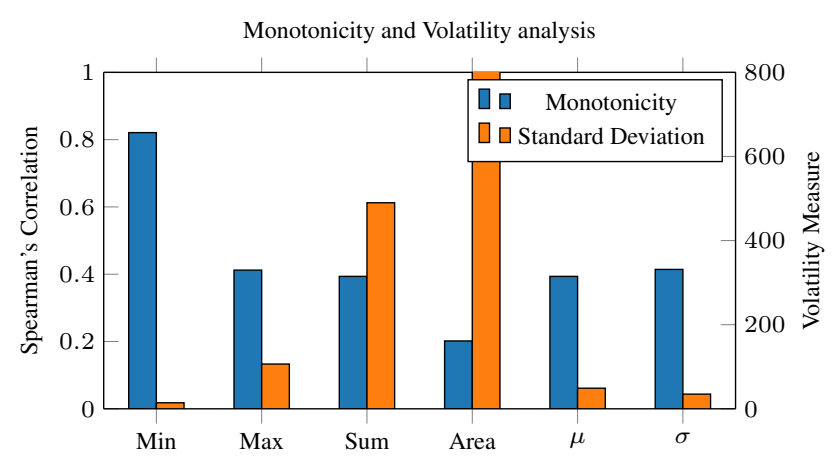

Figure 7. Spearman and volatility analysis

the most monotonic and the least volatile behavior for unhealthy fingers among the parameters, while the sum and the area performed worst. Therefore based on this analysis, our estimated degradation index over discrete time $k$ is defined as:

$$
\tilde{X}(k)=\min _{i, j}\left\{\mathbf{D}_{\mathbf{i j k}}\right\} ; i, j>0, k \geq 0, i \neq j, j>i
$$

The normal operation condition of the hand is found by establishing a pre-specified deterioration threshold $\delta$, so that $0 \leq d(k) \leq \delta$, with $\delta$ a pre-specified threshold. When $d(k)>\delta$, the hand is working under abnormal conditions, requiring further inspection or repair. This threshold has been set based on the empirical observation of the estimated degradation index $\tilde{X}(k)$.

The complete algorithm is briefly described in algorithm 1 for a single motor. The same principle is used to determine the degradation index of the all hand's fingers.

\section{EXPERIMENTAL RESULTS}

\subsection{Offline Evaluation}

The method was tested offline across the entire database, that is the data collected over 50 days of experiments. The estimated degradation index associated to the thumb joints is presented in figure 8 . On the other hand, the results obtained for two healthy fingers are presented in Figure 8. These fingers have been working normally all the time and haven't exhibited any behavior that can indicate a significant degradation.

It is important to note that for the offline tests, about 1000 datapoints were extracted from the database in a random fashion. Concretely, for each individual motor and for each experiment day $E_{d}$, an integer random number $n_{r} \in[1,59000]$ was first generated, in order to extract the next 1000 datapoints starting from $n_{r}$. In other words, the extracted datapoints correspond to the interval $\left[n_{r}, n_{r}+1000\right]$ in the time series, providing thus, time Independence to the proposed method. 


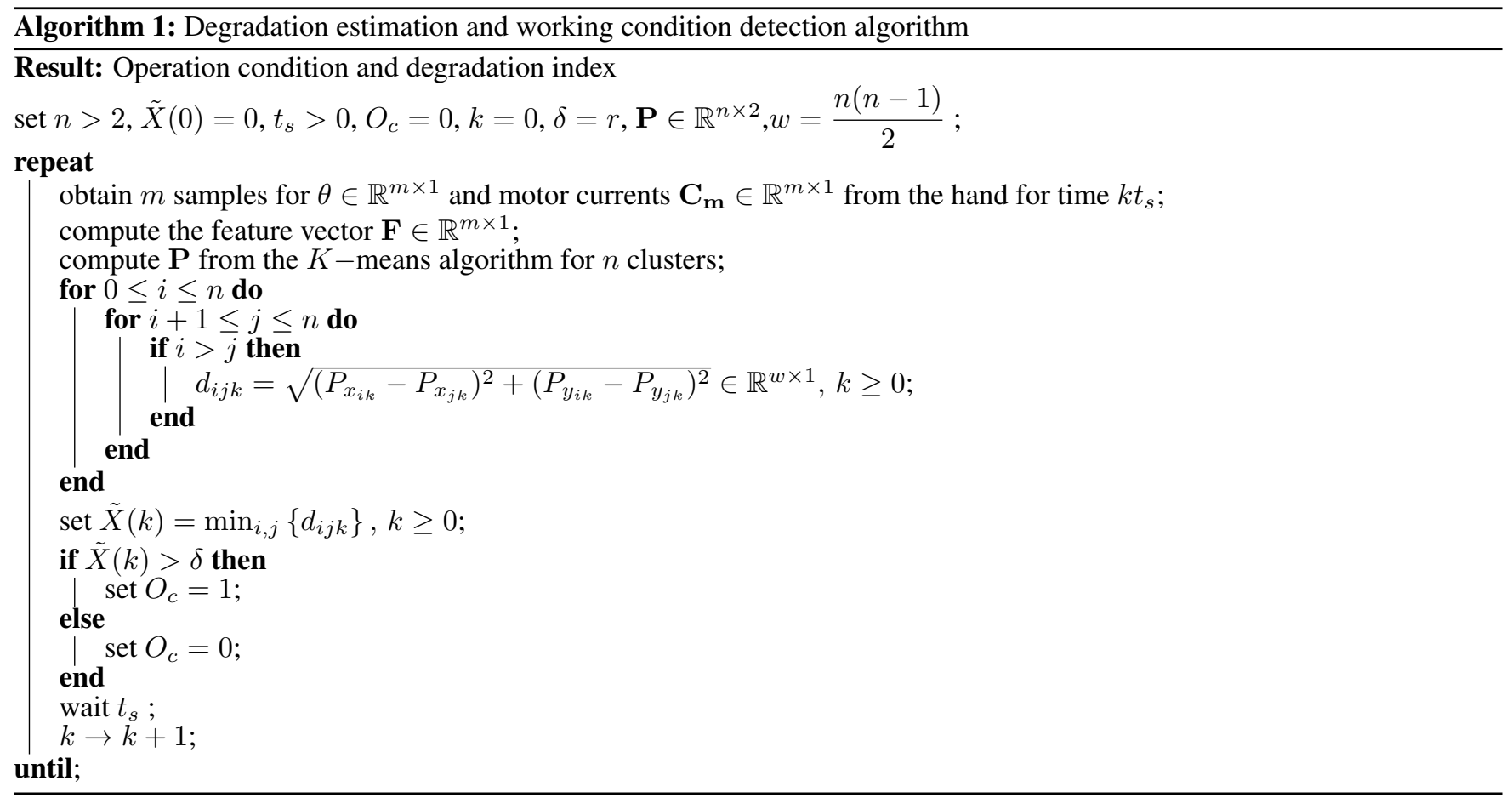

Estimated Degradation Index

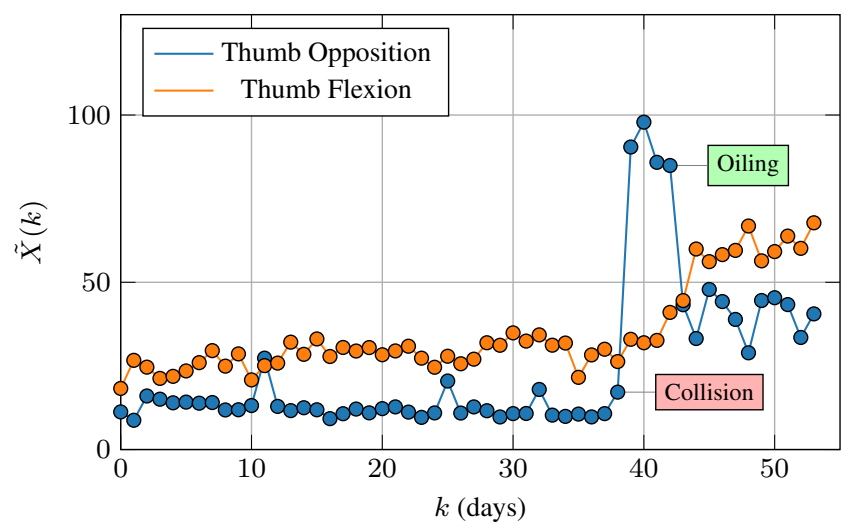

Figure 8. Estimated degradation index for the thumb

\subsection{Online Evaluation}

The method was implemented and tested online during grasping applications. For this purpose, a Kuka IIWA Robot with the Schunk Hand 5-Fingered hand attached was commanded to perform a cyclical grasping task, consisting of the grasping of a test cube from a pick-up point to be transported to a delivery point and backwards, by means of a Thumb-2-Finger grasp (Thumb, Index and Middle fingers). In Figure 10 a diagram of the experimental setup is shown. The estimated degradation algorithm was executed online after the completion of 3 grasps, for which the robot travels to a safe maintenance position away from any contact that the hand could

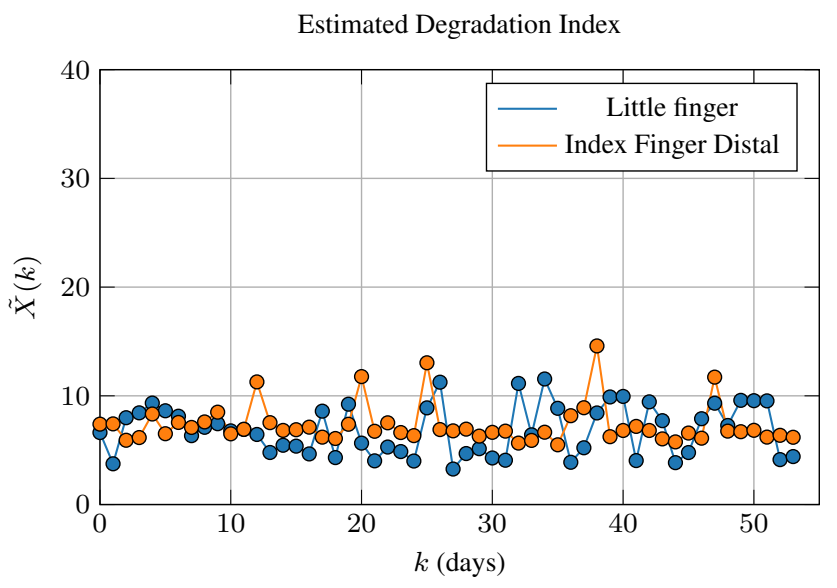

Figure 9. Estimated degradation index for 2 healthy fingers

make with any object. Right after reaching the maintenance position, the hand is commanded to travel in between the limits in absence of grasping loads, time during which the motor currents $C_{m}$ and the angular positions $\theta$ are recorded at a frequency of $100 \mathrm{~Hz}$ using ROS. Lastly, the robot returns to the last delivery point and continues with the cyclical grasping application and the computation of the deterioration is performed in a background parallel task. Here it is important to note, that considering our sensor limitation, the data to feed the model was collected in between grasps to prevent that any contact could be made with any object by the hand, in order to isolate the behavior of the components from external loads and disturbances. 


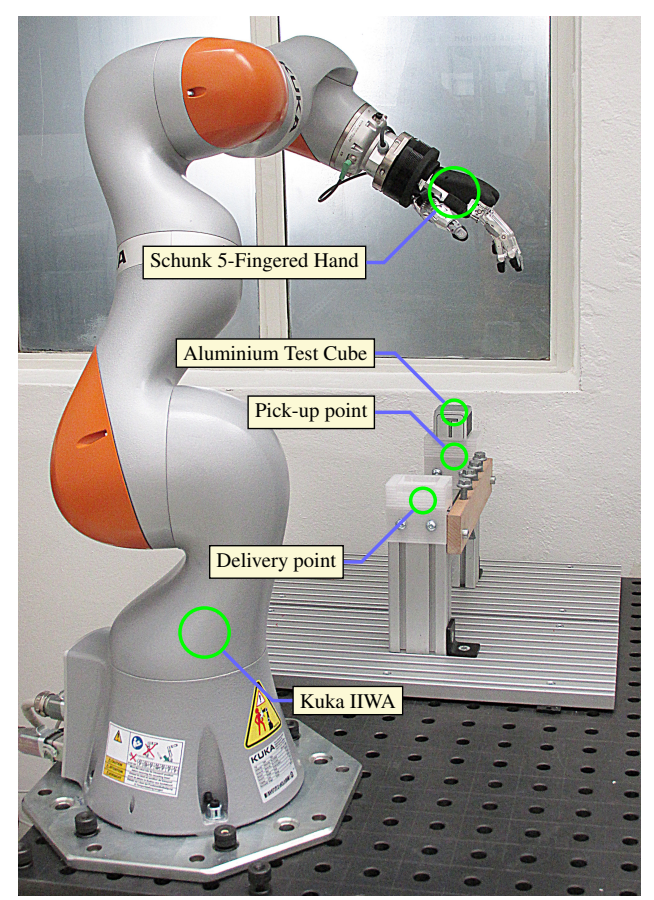

Figure 10. Experimental Setup
According to (Capek, Hughes, \& Warden, 2018), a person looses up to $50 \%$ of the hand function when loosing the thumb, making this finger the most important of the hand. Even though the hand under study is very far from the human hand in every aspect, the thumb of the our robotic hand is also the most critical finger. For this reason, and given our knowledge before hand that the thumb is in a degraded condition, for the online evaluation only the estimated degradation of the thumb is shown in Figure 13. In Figure 11 and 12 the hand appears to be grasping an aluminum and a plastic test cube used for this purpose, performing a Thumb-2-Finger grasp.

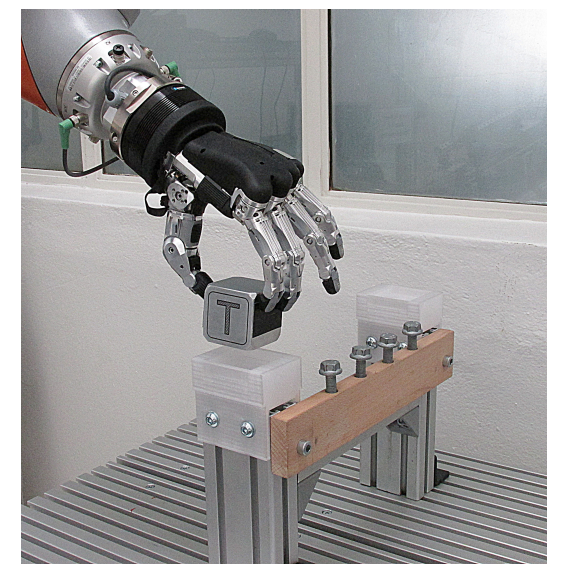

Figure 11. Schunk hand grasping an aluminum test cube during online test

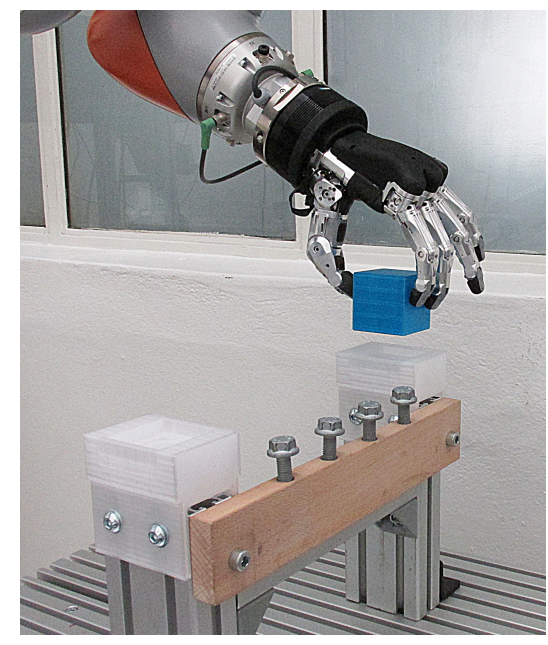

Figure 12. Schunk hand grasping a plastic test cube during online test

Online Estimated Degradation Index

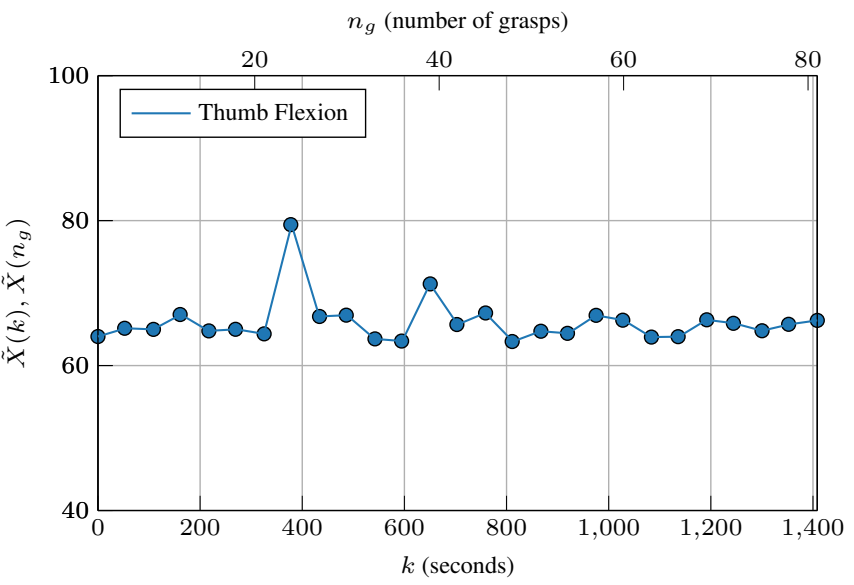

Figure 13. Online estimated degradation index

\section{Discussion}

\subsection{Results}

As it is seen in Figure 8, the estimated degradation index $\tilde{X}$ for the thumb was low for most of the time. However, during the development of a research project, the hand under study collided (day 38 in Figure 8) against parts of the robot it was attached to. This caused a significant crack on a structural screw hole of the hand that prevents it from falling down at the wrist, which makes the hand to be shifted down, causing the structure to be supported by the thumb and in turn, increasing the load on its associated motors and metal-to-metal friction. Our estimated degradation index is consistent with the consequences of this failure as the index increased significantly right after the occurrence of the failure event, as it is seen in Figure 8. Also it is important to note that maintenance actions such as oiling can partially alleviate degraded 
situations, since this particular spot was oiled at the day 42 (Figure 8) in order to prevent further wear and chirping at the finger level due to the increased friction. The degradation index decreased right after oiling as it is observed in Figure 14, however, this action did not recover the degradation to a level prior the collision event. Another consequence of the failure is that it also influenced the dynamics of the surrounding motors, as $\tilde{X}(k)$ of the motor associated to the thumb flexion increases along with $\tilde{X}(k)$ for the motor associated to the thumb opposition. In Figure 15 the place that was oiled is shown.

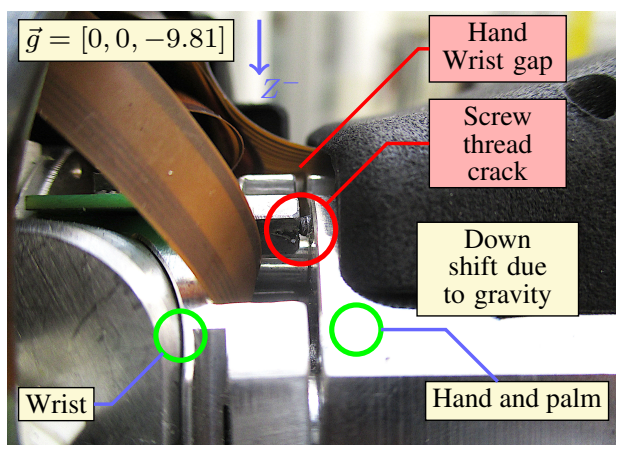

Figure 14. Screw hole crack

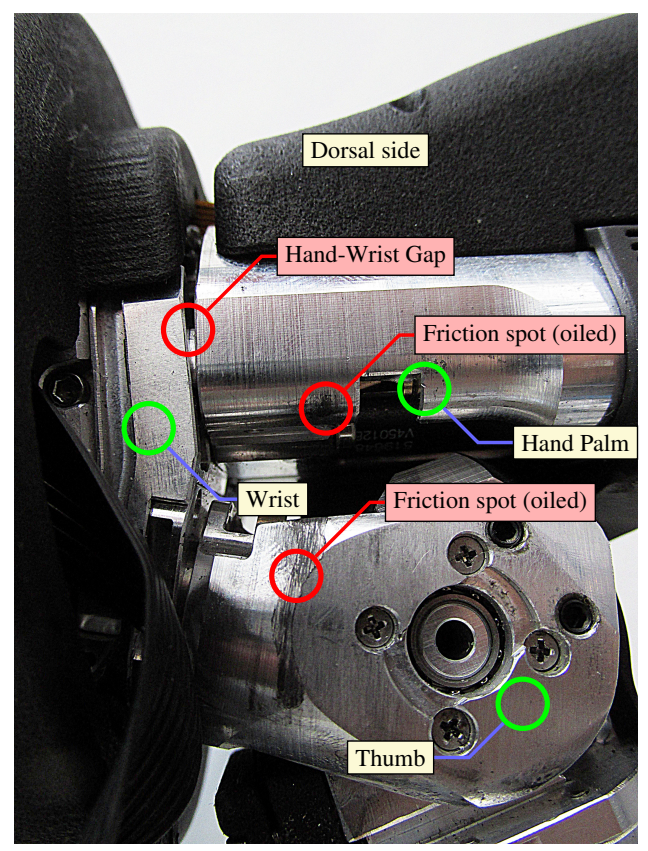

Figure 15. Metal-to-metal friction spot

In Figure 9, the estimated degradation index is shown for 2 different fingers, which have been working under normal behavior. The failure mentioned before has no influence on the dynamics of these fingers, which is also consistent with the Figure 9.

The operation condition of the hand given by the value of $\tilde{X}(k)$ was established based on empirical experience. From the plots of Figure 8 and 9,3 different stages are defined as follows:

1. Normal stage: In this stage the components of the fingers are working normally and there is no need for inspection or further analysis. In this stage the value of $\tilde{X}(k)$ ranges between $0 \leq \tilde{X}(k) \leq 20$.

2. Warning stage: When $20>\tilde{X}(k) \leq 50$, the method indicates that the hand is working under abnormal conditions and the components show some signs of degradation. This stage could also indicate mechanical problems located at the limits of the joints. Preventive maintenance actions such as oiling are recommended.

3. Critical stage: In the critical stage, immediate further inspection and analysis are needed to prevent a rapid deterioration of the system components. In this stage $\tilde{X}(k)>50$ and it could indicate major problems that can lead to catastrophic failures.

In view of Figures 8 and 13, the approach is able to provide consistent results no matter if the method is tested offline using the historical database or online using direct data from grasping applications.

The method proposed in this paper was also compared to the approach presented by (Zheng, 2019). Following his methodology, an estimated degradation index (health index) was computed based on the Hilbert-Huang-Entropy as follows:

$$
H H E=-\sum_{i=1}^{n} p_{i} \ln \left(p_{i}\right)
$$

where $p_{i}$ is defined as:

$$
p_{i}=\frac{h\left(w_{i}\right)}{\sum_{i=1}^{n} h\left(w_{i}\right)}
$$

Here, the term $h\left(w_{i}\right)$ denotes the Marginal Hilbert Spectrum and is defined as follows:

$$
h\left(w_{i}\right)=\int \operatorname{Re}\left\{\sum_{i=1}^{n} A_{i}(t) \exp \left(j \int w_{i}(t) d t\right)\right\}
$$

In this approach, the health index is built upon the envelopes of the signal in Eq. 6, which implies a significant volatility of the signal. Furthermore, since our measured signal spans between $\mathbb{R}^{+}$and $\mathbb{R}^{-}$, an additional conversion is mandatory 
in order to compute the Hilbert-Huang Entropy properly. It turns out, that, unlike the work in (Zheng, 2019), in our case it is the lower envelope of the $H \mathrm{HE}$ that exhibits most monotonic behavior, decreasing immediately after the crash and recovering after the oiling. The Figure 16 depicts the evolution of the HHE based degradation index computed for the Thumb Flexion and its corresponding upper and lower envelopes.

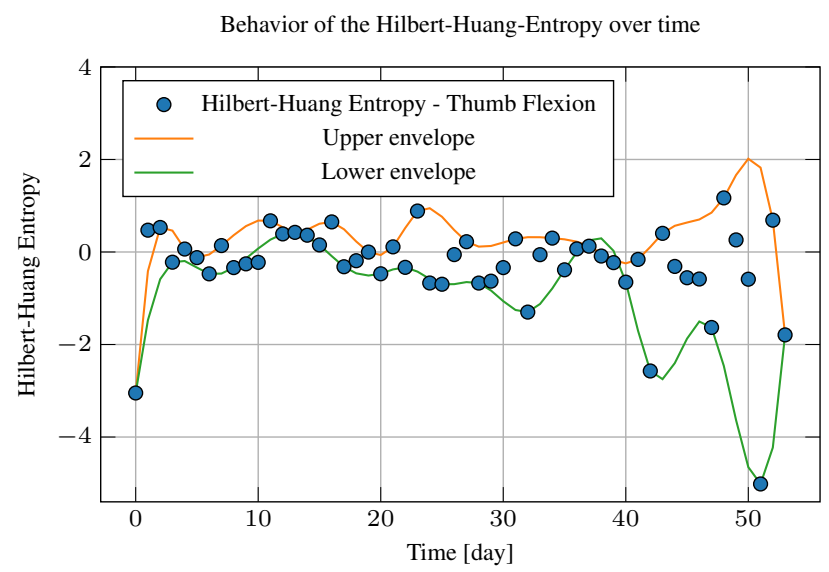

Figure 16. Hilbert-Huang entropy based estimated degradation index

\subsection{Choice of the Number of Clusters}

A key aspect for training the $K$-means algorithm is the choice of the number of clusters, which has a clear relationship with the optimization objective of this algorithm given by:

$$
\min _{\substack{c_{1}, \ldots, c_{m} \\ \mu_{1}, \ldots, \mu_{K}}} J\left(c_{1}, \ldots, c_{m}, \mu_{1}, \ldots, \mu_{K}\right)
$$

with

$$
J=\frac{1}{m} \sum_{i=1}^{m}\left\|x_{i}-\mu_{c_{i}}\right\|^{2}
$$

The Figure 17 shows that the value of $J$ decreases as the number of clusters increases. Using 5 or 6 clusters for the algorithm has shown to have a good balance between performance and accuracy. These are the values we have used during our tests.

\subsection{Limitations}

Given the complexity of the structure of the hand's finger components and the impossibility to measure small parts, it is not possible by this method to identify accurately which component is exhibiting problems or has a high level of degradation, but to detect that the system needs at least inspection or further analysis. Another important issue to remark is that this approach is unlikely to be ported to such robotic hands based on TDMs only using tendon data (tension, displacement, etc.). The reason for this is that the TDMs have

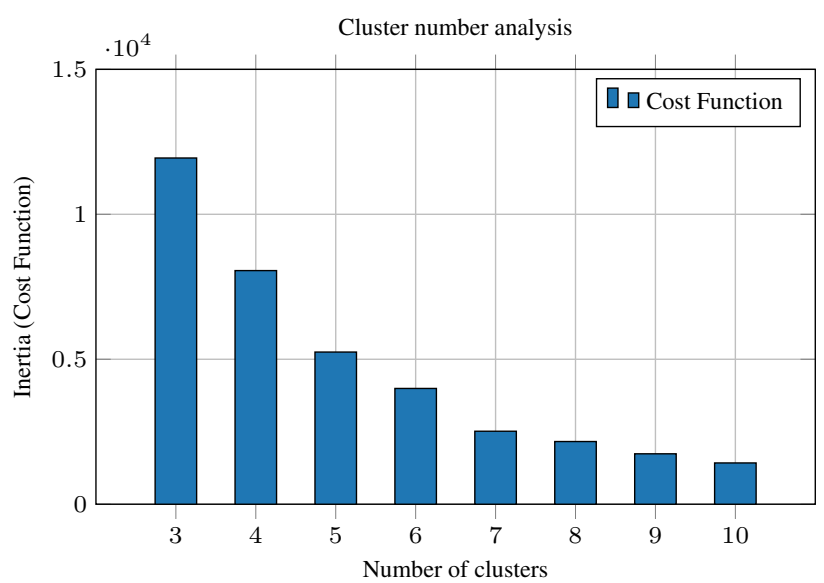

Figure 17. Cluster number analysis

a different nature and dynamics and exhibit phenomena like hysteresis.

Additionally, given the nature of the hand and the limited amount of sensors, this method is not able to perform the estimation of the deterioration during grasping time, where the hand components are subjected to loads, disturbances and phenomena that are inherent to the nature of the grasped objects involved. The reason for this is that it is very difficult to distinguish the grasping event from an event such as a collision or contact with an object. Other type of sensors, such as fingertip force sensors are required to overcome this issue, which are not available for this research.

\section{Conclusion}

The approach for the estimation degradation presented in this paper, allows the online and offline detection of this phenomenon maintaining a low complexity using real data from a real system. According to the best of our knowledge, at the publication moment of this paper, this work represents the first attempt of a PHM approach applied on a real robotic hand. Even though this paper presented both online and offline evaluations of this method, our approach does not require necessarily a large amount of data nor existing datasets for estimating the degradation. Generally the acquisition of such datasets is a difficult task that requires a significant amount of time (Ramasso \& Gouriveau, 2014). During the online tests of the method, between 1200 and 1500 data points were necessary to be collected for computing the estimated degradation index defined in Eq. (5). However, the validation of this method required failure data, which is also difficult to obtain. The failure mentioned in this paper may be recreated by exerting a controlled force on the dorsal side of the hand pointing to the palmar side, largely enough to produce the necessary stress on the structural screw hole shown in Figure 15 , causing the thread hole to be ripped off. The collision suffered by the hand described in section 6.1 happened ac- 
cidentally in a similar manner against a robot. In the case of the abnormal condition depicted in Figure 6, one possible way to recreate the failure might be exerting multiple times a counter force on the index finger while traveling to the closed position, so that is able to produce a higher torque than the torque the associated motor is able to output, and thus forcing the lead screw to move in the opposite direction than the intended one.

From what is seen in Figure 8, the estimated degradation index has correlation and consistency regarding the failure events the hand suffered during the development of the research project. Oiling decreased immediately and consistently the value of the $\tilde{X}(k)$, however it did not solve the situation. It was a palliative solution. Considering the rate of change of $\tilde{X}(k)$ after oiling, such a maintenance action must be well informed and propagated to other engineers and analysts in an industrial setting, since it could lead to ignore a complex failure or postpone an urgent repair or inspection due to the oiling cover-up of the underlying phenomenon, potentially causing catastrophic failure.

Another important remark about this method is its independence of several aspects related to grasping, such as, object properties as mass, shape, texture or geometry and robotic variables, such as speed, acceleration, etc. Additionally, according to (Ertel, Lehmann, Medow, Finkbeiner, \& Meyer, 2014), using the air flow analogously as this paper used the motor current, this method could also be tested on pneumatic driven gripper systems used in the industry.

Our research has open new interesting topics and problems that we want to tackle in the future: RUL estimation of the hand's components, degradation estimation under loads, reconfigurable control and identification of the degrading components. Another important open question that rose from this research is, whether our estimated degradation index from Eq. (5) is equivalent to the real phenomenon given in Eq. (1). At this time, this particular issue needs more research. Additionally, we expect to test our approach on other similar robotic hands or gripper systems to evaluate the transferability.

\section{REFERENCES}

Capek, K. D., Hughes, B. D., \& Warden, G. D. (2018). Functional Sequelae and Disability Assessment. In Total Burn Care (p. 673-678.e1). Elsevier. doi: 10.1016/B978-0-323-47661-4.00063-0

Ertel, W., Lehmann, R., Medow, R., Finkbeiner, M., \& Meyer, A. (2014, January). Model Free Diagnosis of Pneumatic Systems using Machine Learning..

Ficuciello, F. (2019, February). Synergy-Based Control of Underactuated Anthropomorphic Hands. IEEE Transactions on Industrial Informatics, 15(2), 1144-1152. doi: 10.1109/TII.2018.2841043
Garay, J. M., \& Diedrich, C. (2019, September). Remaining Useful Life estimation for production devices in the automotive industry based on health threshold and cyclical data streaming. In 2019 24th IEEE International Conference on Emerging Technologies and Factory Automation (ETFA) (pp. 910-915). Zaragoza, Spain: IEEE. doi: 10.1109/ETFA.2019.8869455

Huynh, K. T., Grall, A., \& Berenguer, C. (2019, March). A Parametric Predictive Maintenance Decision-Making Framework Considering Improved System Health Prognosis Precision. IEEE Transactions on Reliability, 68(1), 375-396. doi: 10.1109/TR.2018.2829771

Kappassov, Z., Corrales, J.-A., \& Perdereau, V. (2015, December). Tactile sensing in dexterous robot hands - Review. Robotics and Autonomous Systems, 74, 195-220. doi: 10.1016/j.robot.2015.07.015

Khelif, R., Chebel-Morello, B., Malinowski, S., Laajili, E., Fnaiech, F., \& Zerhouni, N. (2017, March). Direct Remaining Useful Life Estimation Based on Support Vector Regression. IEEE Transactions on Industrial Electronics, 64(3), 2276-2285. doi: 10.1109/TIE.2016.2623260

Lei, Y., Li, N., \& Lin, J. (2016, December). A New Method Based on Stochastic Process Models for Machine Remaining Useful Life Prediction. IEEE Transactions on Instrumentation and Measurement, 65(12), 2671-2684. doi: 10.1109/TIM.2016.2601004

Loutas, T. H., Roulias, D., \& Georgoulas, G. (2013, December). Remaining Useful Life Estimation in Rolling Bearings Utilizing Data-Driven Probabilistic E-Support Vectors Regression. IEEE Transactions on Reliability, 62(4), 821-832. doi: 10.1109/TR.2013.2285318

Patil, M. A., Tagade, P., Hariharan, K. S., Kolake, S. M., Song, T., Yeo, T., \& Doo, S. (2015, December). A novel multistage Support Vector Machine based approach for Li ion battery remaining useful life estimation. Applied Energy, 159, 285-297. doi: 10.1016/j.apenergy.2015.08.119

Peng, Y., Cheng, F., Qiao, W., \& Qu, L. (2017, May). Fault prognosis of drivetrain gearbox based on a recurrent neural network. In 2017 IEEE International Conference on Electro Information Technology (EIT) (pp. 593-599). Lincoln, NE, USA: IEEE. doi: 10.1109/EIT.2017.8053433

Ramasso, E., \& Gouriveau, R. (2014, June). Remaining Useful Life Estimation by Classification of Predictions Based on a Neuro-Fuzzy System and Theory of Belief Functions. IEEE Transactions on Reliability, 63(2), 555-566. doi: 10.1109/TR.2014.2315912

Rodrigues, L. R. (2018, February). Remaining Useful Life Prediction for Multiple-Component Systems Based on a System-Level Performance Indicator. IEEE/ASME Transactions on Mechatronics, 23(1), 141-150. doi: 
10.1109/TMECH.2017.2713722

ROS Driver, S. H. S. (Last access: 202005-16 01:02:10). Schunk_svh_driver ROS. http://wiki.ros.org/schunk_svh_driver.

Ruehl, S. W., Parlitz, C., Heppner, G., Hermann, A., Roennau, A., \& Dillmann, R. (2014, December). Experimental evaluation of the schunk 5-Finger gripping hand for grasping tasks. In 2014 IEEE International Conference on Robotics and Biomimetics (ROBIO 2014) (pp. 2465-2470). Bali, Indonesia: IEEE. doi: 10.1109/ROBIO.2014.7090710

Schunk Hand SVH Assembly and Operating Manual, S. G. (Last access: 2020-05-16 00:56:44). Schunk Hand SVH Assembly and Operating Manual. https://schunk.com/filead$\mathrm{min} / \mathrm{pim} /$ docs/IM0020890.PDF.

Si, X.-S., Zhang, Z.-X., \& Hu, C.-H. (2017). Estimating RUL with Three-Source Variability in Degradation Modeling. In Data-Driven Remaining Useful Life Prognosis Techniques (pp. 143-180). Berlin, Heidelberg: Springer Berlin Heidelberg. doi: 10.1007/978-3662-54030-5_6

Suehiro, K., Ozawa, R., \& Van Heerden, K. (2017, October). Force-Sensorless Fault Tolerant Detection and Switching Control of Tendon-Driven Mechanisms With Redundant Tendons. IEEE Robotics and Automation Letters, 2(4), 2248-2254. doi: 10.1109/LRA.2017.2724771

Tobon-Mejia, D. A., Medjaher, K., Zerhouni, N., \& Tripot,
G. (2012, June). A Data-Driven Failure Prognostics Method Based on Mixture of Gaussians Hidden Markov Models. IEEE Transactions on Reliability, 61(2), 491-503. doi: 10.1109/TR.2012.2194177

Walck, G., Haschke, R., Meier, M., \& Ritter, H. J. (2017, September). Robot self-protection by virtual actuator fatigue: Application to tendon-driven dexterous hands during grasping. In 2017 IEEE/RSJ International Conference on Intelligent Robots and Systems (IROS) (pp. 2200-2205). Vancouver, BC: IEEE. doi: 10.1109/IROS.2017.8206039

Walker, R., De La Rosa, A., Elias, H., Godden, M., \& Goldsmith, J. (2010, December). Advances in actuation technology for compliant Dextrous manipulation. In 2010 IEEE International Conference on Robotics and Biomimetics (pp. 1429-1433). doi: 10.1109/ROBIO.2010.5723539

Wang, Z.-Q., Hu, C.-H., \& Fan, H.-D. (2018, February). Real-Time Remaining Useful Life Prediction for a Nonlinear Degrading System in Service: Application to Bearing Data. IEEE/ASME Transactions on Mechatronics, 23(1), 211-222. doi: 10.1109/TMECH.2017.2666199

Zheng, Y. (2019, February). Predicting Remaining Useful Life Based on Hilbert-Huang Entropy with Degradation Model. Journal of Electrical and Computer Engineering, 2019, 1-11. doi: 10.1155/2019/3203959 